\title{
Distinct immunophenotypes and prognostic factors in renal cell carcinoma with sarcomatoid differentiation: a systematic study of 19 immunohistochemical markers in 42 cases
}

\author{
Wenjuan Yu', Yuewei Wang ${ }^{3}$, Yanxia Jiang ${ }^{1}$, Wei Zhang ${ }^{2^{*}}$ and Yujun $\mathrm{Li}^{1^{*}}$
}

\begin{abstract}
Background: Renal cell carcinoma (RCC) with sarcomatoid differentiation is a relatively rare tumor containing both carcinoma and sarcomatoid components. However, there has not been a systemic study on immunophenotypes of renal cell carcinoma with sarcomatoid differentiation, especially using some renal specific immunohistochemical markers. In this study, we aimed to comprehensively investigate the distinct immunophenotypes of RCC with sarcomatoid differentiation

to analyze the pathogenesis of sarcomatoid differentiation and identify new prognostic factors in RCC with sarcomatoid differentiation.

Methods: A total of 42 cases of RCCs with sarcomatoid differentiation were enrolled into the study. Immunohistochemistry study was performed on tissue microarrays to evaluate the expressions of 19 immunohistochemical markers including a series of epithelial, mesenchymal markers and RCC specific markers. Kaplan-Meier method was applied to assess the prognostic values of CD10, CAIX, p53 and BCl-2.

Results: Histologically, 42 cases of RCCs with sarcomatoid differentiation presented with different proportions of carcinoma and sarcomatoid components. The cohort contained 35 cases of clear cell renal cell carcinoma (CCRCC) and 7 cases of chromophobe renal cell carcinoma (ChRCC) based on the carcinoma components. Immunohistochemically, all cases were positive for vimentin, and 80\% of cases showed immunostaining for at least one epithelial marker, such as CK, EMA, CK7 and CK18. Notably, the expression rates of CAIX, CD10 and PAX8 in sarcomatoid cells were $76 \%, 76 \%$ and $64 \%$, respectively. The carcinoma component of the tumors showed differentient labeling for CAIX, CD10, vimentin, CK7 and CD117 in CCRCC vs ChRCC, but the sarcomatoid component lost the specificity for these markers $(p<0.05)$. Patients with positive expressions of CAIX, p53 and $\mathrm{BCl}-2$ had a poor prognosis.

(Continued on next page)
\end{abstract}

\footnotetext{
*Correspondence: zhangwei686538@126.com; wenjuan801023@163.com; liyujun_66@163.com

2Department of Pathology, 401 Hospital of People's Liberation Army, 22 Minjiang Rd, Qingdao 266071, China

'Department of Pathology, The Affiliated Hospital of Qingdao University, 16

Jiangsu Road, Qingdao 266003, China

Full list of author information is available at the end of the article
} 
(Continued from previous page)

Conclusions: The sarcomatoid cells in RCC with sarcomatoid differentiation express both epithelial and mesenchymal markers, supporting their epithelial origin. PAX8, CAIX and CD10 could be used as the reliable and useful markers to determine the renal origin of sarcomatoid cells such as in fine needle aspiration cases and metastatic RCC with sarcomatoid differentiation. CAIX, p53 and BCl-2 might play important roles in the transformation from renal cell carcinoma to high malignant sarcomatoid differentiation, and these three immunohistochemical markers are adverse prognostic factors for the survival of patients with RCC with sarcomatoid differentiation.

Keywords: Renal cell carcinoma, Sarcomatoid, Pathogenesis, Immunohistochemistry

\section{Background}

Renal cell carcinoma (RCC) with sarcomatoid differentiation is a rare renal carcinoma, also called sarcomatiod renal cell carcinoma, spindle cell carcinoma and carcinomatous sarcoma, etc. One of its key histological features is the presence of both carcinoma and sarcomatoid differentiation in the tumor [1]. According to the 2004 and 2016 WHO Classification of Tumors of the Urinary System and Male Genital Organs, RCC with sarcomatoid differentiation is not recognized as a separate and distinct entity and the sarcomatoid component could arise from the genetic background of any of the RCC subtypes [2]. RCC with sarcomatoid differentiation presents unique histological characteristics, invasive behavior and poor prognosis [3]. Due to the low morbidity and the deficiency of study data, the pathogenesis of the transformation from carcinoma to sarcomatoid component along with the prognostic factors for RCC with sarcomatoid differentiation remains unelucidated. Herein we performed our immunohistological study using 19 immune markers, including the renal specific markers PAX8 and CAIX in 42 cases of RCCs with sarcomatoid differentiation. Our study was focused on the analysis of the expression differences between those two components. This study may shed some light on revealing the pathogenesis and assessing the prognosis of the patients with this relatively rare renal carcinoma.

\section{Methods}

Among the 42 cases of RCCs with sarcomatoid differentiation, 35 cases were collected in the Affiliated Hospital of Qingdao University and 7 cases were collected in 401 Hospital of People's Liberation Army from November 2003 to January 2015. All cases were re-diagnosed by two pathologists majored in Genitourinary Pathology. The samples were made into 2 tissue microarrays and there were 42 punches in each tissue microarray. Each tumor was made into 2 punches including carcinoma and sarcomatoid components separately based on the observation under microscope. The diameter of the cores was $2 \mathrm{~mm}$.

All tissues were 4-um-thick, fixed with $4 \%$ neutral formaldehyde, and embedded in paraffin for $\mathrm{H} \& \mathrm{E}$ staining. Immunohistochemistry was performed on tissue microarrays comprising of 42 cases containing both typical renal cell carcinoma cells and sarcomatoid cells. Primary antibodies used in the study including vimentin (Cell Marque, V9), cytokeratin (CK) (Origene, AE1/ $\mathrm{AE} 3$ ), epithelial membrane antigen (EMA) (Thermo, E29), cytokeratin-7 (CK7) (Cell Marque, OV-TL12/30), cytokeratin-18 (CK18) (Origene, UMAB50), high molecular weight cytokeratin (HMW-CK) (Cell Marque, CKHMW), and CAIX (Novus, polyclonal), CD10 (Ventana, SP67), PAX8 (Ventana, MRQ-50), alpha-methylacylCoA racemase (AMACR) (Zeta,13H4), CD117 (Ventana, 9.7), CD99 (Covance, O13), SMA (Origene, 1A4), CD34 (Leica, OBEnd/10), S100 (Thermo, 4c4.9), Melanoma (Cell Marque, HMB45), and Melan A (Cell Marque, A103), p53 (Ventana, DO-7), and Bcl-2 (Thermo, 8c8). UltraView $\mathrm{DAB}$ detection kit was purchased from Ventana (Arizona, America). All immunohistochemistry assays were performed on the Roche BenchMark XT fully automatic IHC/ISH instrument by optimized protocols. Positive and negative controls were used in this study.

The positive staining of vimentin, CK, CK7, CK18, HMW-CK, CD34, AMACR, SMA, Bcl-2, HMB45 and melan A predominantly appeared in the tumor cell cytoplasm, whereas p53 and PAX8 mainly appeared in the nuclei. CD99, EGFR and CAIX tended to express on the cell membrane. EMA, CD10 and CD117 expressed on the cell membrane or cytoplasm; S100 expressed in the cytoplasm and nuclei. The positive staining was first scored as $0,1,2,3$ based on the staining intensity (no staining, faint, mild, strong) and the percentage of positively stained cells was $(0 \%, \leq 25 \%, 26 \% \sim 75 \%,>75 \%)$, respectively. Two scores then multiplied together, which were considered as the final scores, grading as $-(0-1)$, $1+(2-4), 2+(5-6), 3+(7-9)$.

\section{Clinical follow-up and survival analysis}

Follow-up data were collected for 33 cases. Survival was calculated from the date of surgery to the death or the last follow-up visit. For the analysis, only deaths for RCCs with sarcomatoid differentiation were considered as events. Survival curves were derived from Kaplan-Meier analysis and log-rank test to compare overall survival 
between different groups based on the expressions of CD10, CAIX, p53 and Bcl-2 since the four markers might play roles in the proliferation of tumors. Furthermore, in order to investigate the factors that may affect survival patterns, the $P$ values for prognostic factors analyses were adjusted for multiple analyses using Cox regression model.

\section{Statistical analysis}

SPSS 13.0 software was applied to perform statistical analysis. Statistical significance was tested by chi-square test (or Fisher's exact test) to compare each marker's expression in the carcinoma and sarcomatoid components. Statistical significance was considered as $P$ value less than 0.05 .

\section{Results}

There were 35 clear cell renal cell carcinomas (CCRCCs) and 7 chromophobe renal cell carcinomas (ChRCCs) based on the carcinoma component. The sarcomatoid components of the investigated tumors resembled these entities as fibrosarcoma, leiomyosarcoma and malignant fibrous histiocytoma (Fig. 1a-d).

The immunohistochemistry results of the two components including carcinoma and sarcomatoid cells were listed in Table 1. The cohorts could be divided into four groups based on the expression panels of each immunohistochemical markers: carcinoma positive and sarcomatoid cell positive $(\mathrm{C}+/ \mathrm{S}+)$; carcinoma positive and sarcomatoid cell negative $(\mathrm{C}+/ \mathrm{S}-)$; carcinoma negative and sarcomatoid cell positive $(\mathrm{C}-/ \mathrm{S}+)$; carcinoma negative and sarcomatoid cell negative $(\mathrm{C}-\mathrm{S} / \mathrm{S}-)$. On the whole, the expressions of vimentin, EMA, SMA, and Bcl-2 were significantly different between carcinoma and sarcomatoid cells $(P<0.05)$. No significant difference was revealed in the expressions of other immunohistochemical markers between the two components $(P>0.05)$. The following is the detailed results.

\section{The expression patterns of vimentin, CK, and EMA in RCCs with sarcomatoid differentiation}

The positive expression rates of vimentin were $52 \%(22 / 42)$ in carcinoma cells and $100 \%(42 / 42)$ in sarcomatoid cell $(P=0.000)$. The main expression panels of vimentin were $\mathrm{C}+/ \mathrm{S}+(22 / 42)$ and $\mathrm{C}-/ \mathrm{S}+(20 / 42)$. The positive expression rates of $\mathrm{CK}$ in carcinoma and sarcomatoid cells were $81 \%$ $(34 / 42)$ and $50 \%(21 / 42)$, respectively $(P=0.005)$. The main expression panels of $\mathrm{CK}$ were $\mathrm{C}+/ \mathrm{S}+(18 / 42)$ and $\mathrm{C}+/ \mathrm{S}-(16 / 42)$. The positive expression rates of EMA were $88 \%(37 / 42)$ and $50 \%(21 / 42)$ in carcinoma and sarcomatoid cells, respectively $(P=0.000)$. The expression panels of EMA were $\mathrm{C}+/ \mathrm{S}+(21 / 42)$ and $\mathrm{C}+\mathrm{S}-(16 / 42)$.

\section{The expression patterns of CK7, CK18, and HMW-CK in RCC with sarcomatoid differentiation}

CK7 was expressed in carcinoma cells in 11 of 42 cases $(11 / 42)$ and sarcomatoid cells in 5 of 42 cases $(5 / 42)$ $(P=0.164)$. The expression panels of CK7 were $\mathrm{C}+/ \mathrm{S}$ -

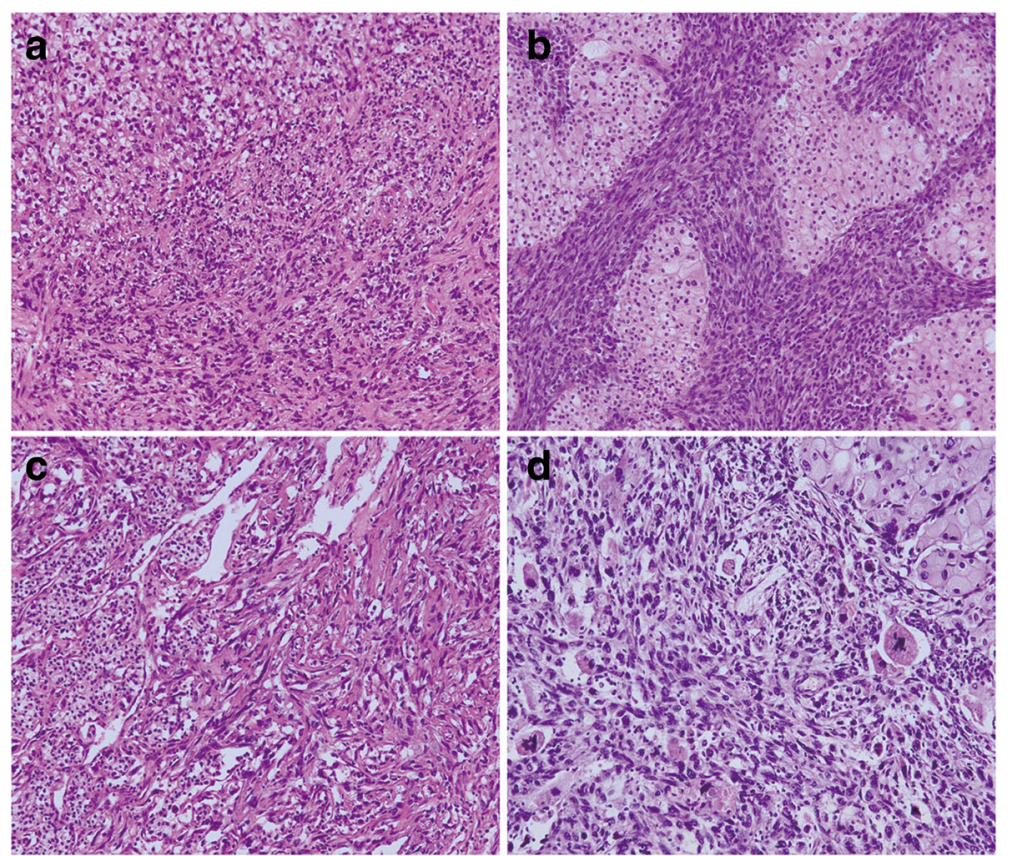

Fig. 1 The histopathological figures of RCC with sarcomatoid differentiation. a CCRCC together with fibrosarcomatoid cells $\mathbf{b}$ ChRCC together with fibrosarcomatoid cells c Clear cell carcinoma together with leiomyosarcoma-like cells $\mathbf{d}$ ChRCC together with malignant fibrous histiocytoma-like cells. H\&E $\times 100$ 
Table 1 The immunophenotypes of 42 cases of RCCs with sarcomatoid differentiation and the expression differences between carcinoma and sarcomatoid differentiation

\begin{tabular}{|c|c|c|c|c|c|c|c|}
\hline Markers & $\mathrm{C}+/ \mathrm{S}+$ & $\mathrm{C}+/ \mathrm{S}-$ & $\mathrm{C}-/ \mathrm{S}+$ & C-/S- & Total C+ & Total S+ & $P$ value \\
\hline Vimentin & 22 & 0 & 20 & 0 & $22(52 \%)$ & $42(100 \%)$ & $0.000^{*}$ \\
\hline CK & 18 & 16 & 3 & 5 & 34 (81\%) & $21(50 \%)$ & $0.005^{*}$ \\
\hline EMA & 21 & 16 & 0 & 5 & 37 (88\%) & $21(50 \%)$ & $0.000^{*}$ \\
\hline CK7 & 0 & 11 & 5 & 26 & $11(26 \%)$ & $5(12 \%)$ & 0.164 \\
\hline CK18 & 12 & 13 & 7 & 10 & $25(60 \%)$ & 19 (45\%) & 0.275 \\
\hline HMW-CK & 0 & 0 & 3 & 39 & $0(0 \%)$ & $3(7 \%)$ & 0.241 \\
\hline CAIX & 20 & 1 & 12 & 9 & $21(50 \%)$ & 32 (76\%) & $0.023^{*}$ \\
\hline CD10 & 20 & 3 & 12 & 7 & 23 (55\%) & 32 (76\%) & 0.065 \\
\hline PAX8 & 24 & 10 & 3 & 4 & 34 (81\%) & 27 (64\%) & 0.141 \\
\hline AMACR & 3 & 8 & 3 & 28 & $11(26 \%)$ & $6(14 \%)$ & 0.277 \\
\hline CD117 & 0 & 7 & 3 & 32 & $7(17 \%)$ & $3(7 \%)$ & 0.313 \\
\hline CD99 & 0 & 3 & 9 & 30 & $3(7 \%)$ & $9(21 \%)$ & 0.116 \\
\hline SMA & 0 & 0 & 8 & 34 & $0(0 \%)$ & $8(19 \%)$ & $0.005^{*}$ \\
\hline CD34 & 0 & 0 & 0 & 42 & $0(0 \%)$ & $0(0 \%)$ & - \\
\hline S100 & 0 & 0 & 0 & 42 & $0(0 \%)$ & $0(0 \%)$ & - \\
\hline HMB45 & 0 & 0 & 0 & 42 & $0(0 \%)$ & $0(0 \%)$ & - \\
\hline MelanA & 0 & 0 & 0 & 42 & $0(0 \%)$ & $0(0 \%)$ & - \\
\hline P53 & 8 & 2 & 12 & 20 & $10(24 \%)$ & $20(48 \%)$ & $0.040^{*}$ \\
\hline $\mathrm{BCl}-2$ & 9 & 16 & 0 & 17 & $25(60 \%)$ & $9(21 \%)$ & $0.001^{*}$ \\
\hline
\end{tabular}

C carcinoma cell, $S$ sarcomatoid cell

*The difference is statistically significant

(11/42) and C-/S+ (5/42). CK7 was not simultaneously expressed in two types of cells. CK18 was expressed in carcinoma cells in 25 of 42 cases (25/42) and sarcomatoid cells in 19 of 42 cases $(19 / 42)(P=0.275)$. The main expression panels of CK18 were $\mathrm{C}+/ \mathrm{S}_{+}(12 / 42)$ and $C+/ S-(13 / 42)$. HMW-CK was only expressed in the sarcomatoid cells in 3 of 42 cases $(3 / 42)(P=0.241)$.

\section{The expression patterns of CAIX, CD10, and PAX8 in RCC} with sarcomatoid differentiation

The positive expression rates of CAIX in carcinoma and sarcomatoid cells were 50\% (21/42) and 76\% (32/42), respectively $(P=0.023)$. The major expression panels of CAIXwere $\mathrm{C}+/ \mathrm{S}_{+}(20 / 42)$ and $\mathrm{C}-/ \mathrm{S}_{+}$(12/42). Among them, CAIX was diffusely strong expressed (3+) in carcinoma cells in 18 of 42 cases and in sarcomatoid cells in 29 of 42 cases. The positive expression rates of CD10 in carcinoma and sarcomatoid cells were 55\% (23/ $42)$ and $76 \%(32 / 42)$, respectively $(P=0.065)$. The main expression panels of CD10 were $\mathrm{C}+/ \mathrm{S}+(20 / 42)$ and $\mathrm{C}-/ \mathrm{S}$ $+(12 / 42)$. PAX8 was expressed in carcinoma cells in 34 of 42 cases $(34 / 42)$ and sarcomatoid cells in 11 of 42 cases (27/42) $(P=0.141)$. Notably, among them, 24 cases showed co-expression of PAX8 in two types of cells (24/42). The main expression panels of PAX8 were C
$+/ \mathrm{S}+(24 / 42)$, and $\mathrm{C}+/ \mathrm{S}-(10 / 42)$. The positive expressions of CAIX, CD10, and PAX8 were showed in Fig. 2a, b, and c.

\section{The expression patterns of AMACR, CD117, and CD99 in RCC with sarcomatoid differentiation \\ The expression panels of AMACR were $\mathrm{C}+\mathrm{S}+(3 / 42)$, $\mathrm{C}+/ \mathrm{S}-(8 / 42)$, and $\mathrm{C}-/ \mathrm{S}+(3 / 42)$. The expression pat- terns showed no difference between carcinoma and sarcomatoid cells $(P=0.277)$. CD117 were merely expressed in carcinoma cells in 7 of 42 cases and sar- comatoid cells in 3 of 42 cases $(P=0.313)$. In addition, 3 samples presented CD99 expression in carcinoma cells and 9 cases presented CD99 expression in sarcomatoid cells $(P=0.116)$.}

The expression patterns of SMA, CD34, S100, HMB45, and melanA in RCC with sarcomatoid differentiation

SMA was expressed in sarcomatoid cells in 8 cases without positive expression in carcinoma cells $(P=0.005)$. But beyond that, CD34, S100, HMB45 and melanA were not expressed in two types of cells in all cases.

\section{The expression patterns of p53 and $\mathrm{BCl}-2$ in RCC with sarcomatoid differentiation}

Ten cases showed p53 expression in carcinoma cells and 20 cases showed p53 expression in sarcomatoid cells $(P=0.04)$. The main expression panels of $\mathrm{p} 53$ were $\mathrm{C}+/ \mathrm{S}+$ $(8 / 42)$ and $\mathrm{C}-/ \mathrm{S}+(12 / 42)$. The positive expression rates of Bcl-2 in carcinoma and sarcomatoid cells were 60\% (25/ $42)$ and $21 \%(9 / 42)$, respectively $(P=0.001)$. The expression panels of Bcl-2 were $\mathrm{C}+/ \mathrm{S}+(9 / 42)$ and $\mathrm{C}+/ \mathrm{S}-$ $(16 / 42)$. The positive expression of $\mathbf{p} 53$ were showed in Fig. 2d.

Expression differences of CAIX, CD10, vimentin, CK7 and CD117 in the carcinoma and sarcomatoid cells between CCRCC and ChRCC

The expressions of CAIX, CD10, vimentin, CK7 and CD117 in the carcinoma component between CCRCC and ChRCC showed significant differences $(P<0.05)$. However, no significant difference was revealed in the sarcomatoid component between the two tumors $(P>0.05)$ (Table 2).

\section{Clinical follow-up and survival analysis}

Follow-up data were collected for 33 cases with the follow-up time ranged from 1 to 42 months. Among them, 1 patient died from other disease rather than tumor. There were another 24 deaths, including 22 CCRCCs and 2 ChRCCs according to the carcinoma elements which occurred in the 1st $\sim 30$ th month after the surgery for metastasis of tumors to the bone or lung. Other 8 patients are still alive uneventfully after surgery. 


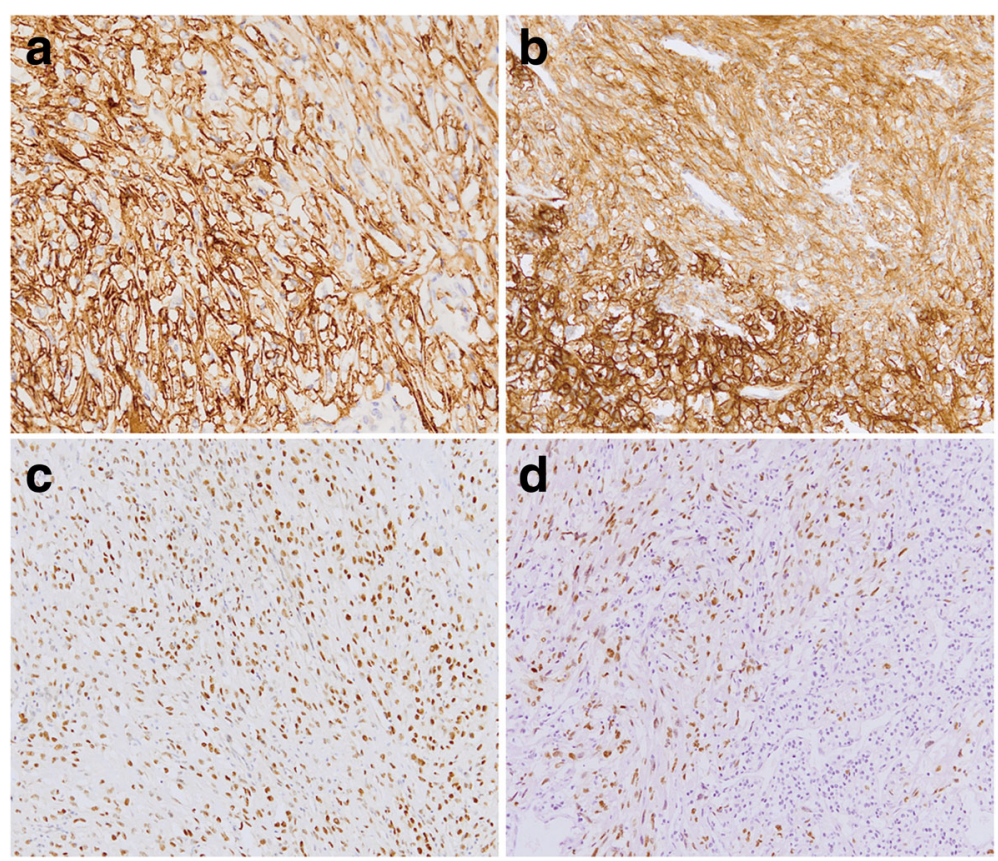

Fig. 2 Immunohistochemical staining figures of RCC with sarcomatoid differentiation. a The positive expression of CA IX in clear cell carcinoma together with fibrosarcomatoid cells $\mathbf{b}$ The positive expression of CD10 in clear cell carcinoma together with fibrosarcomatoid cells $\mathbf{c}$ The positive expression of PAX8 in ChRCC together with fibrosarcomatoid cells $\mathbf{d}$ The positive expression of p53 in fibrosarcomatoid cells and negative expression in clear cell carcinoma. Immunohistochemistry $\times 100$

The Kaplan-Meier survival curves comparing overall survival according to the expressions of CD10, CAIX, p53 and $\mathrm{Bcl}-2$ were presented in Fig. 3. Univariate analysis showed that the expressions of CAIX $(p=0.000)$, p53 $(p=0.000)$ and $\mathrm{Bcl}-2(p=0.001)$ were all adverse prognostic factors for tumor related survival (Fig. 3). Furthermore, the multiple analyses using the Cox regression model revealed that CAIX, p53 and Bcl-2 were independent predictive factors for prognosis of RCC with sarcomatoid differentiation $(P=0.001,0.011,0.013$, respectively).

\section{Discussion}

A malignant tumor comprising of malignant epithelial cells together with mesenchymal cells is called

Table 2 The expression differences of CAIX, CD10, vimentin, CK7 and CD117 between CCRCC and ChRCCs in the carcinoma and sarcomatoid differentiation

\begin{tabular}{lllllll}
\hline & CCRCC & ChRCC & $P$ value & CCRCC & ChRCC & $P$ value \\
& C+ & C+ & & S+ & S+ & \\
\hline CAIX & $23 / 35$ & $0 / 7$ & $0.002^{*}$ & $29 / 35$ & $7 / 7$ & 0.567 \\
CD10 & $21 / 35$ & $0 / 7$ & $0.009^{*}$ & $27 / 35$ & $5 / 7$ & 1.000 \\
Vimentin & $23 / 35$ & $0 / 7$ & $0.002^{*}$ & $35 / 35$ & $7 / 7$ & - \\
CK7 & $3 / 35$ & $7 / 7$ & $0.000^{*}$ & $5 / 35$ & $0 / 7$ & 0.569 \\
CD117 & $3 / 35$ & $6 / 7$ & $0.000^{*}$ & $2 / 35$ & $0 / 7$ & 1.000 \\
\hline
\end{tabular}

$C$ carcinoma cell, $S$ sarcomatoid cell

*The difference is statistically significant carcinosarcoma. According to the histological classification of 2016 WHO for the epithelial tumors of kidney, this type of tumor is regarded as transformation from different types of renal cell carcinoma, rather than an independent histological entity [2]. However, a large sample and systematic study on immunophenotypes of both carcinoma and sarcomatoid cells are still limited, especially for some renal specific immunohistochemical markers [4]. Herein we performed immunohistochemistry study to characterize 19 different markers in 42 cases of RCC with sarcomatoid differentiation and analyzed the expression patterns of these markers in both carcinoma and sarcomatoid cells. We grouped these immunohistochemical markers into 4 subgroups as followed based on their different physiological functions.

\section{Epithelial markers}

In the present study, sarcomatoid cells in $81 \%$ cases expressed at least one epithelial marker. CK and EMA were expressed in approximately $62 \%$ cases. Sarcomatoid cells in 19 cases expressed CK18. However, 8 cases presented no expression of any tested epithelial marker. 5 cases showed CK7 positive staining in sarcomatoid cells rather than in clear cell carcinomas. The expressions of epithelial markers in sarcomatoid cells confirmed that sarcomatoid cells resulted from different differentiation orientations at different degrees of epithelial cells. Thus we recommend a series of cytokeratins with different 
CD10 expression

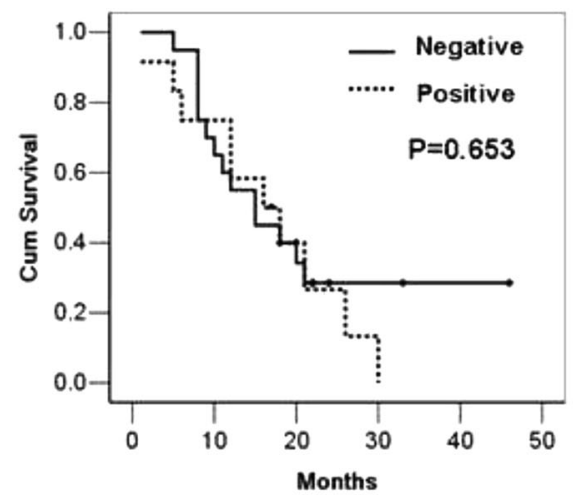

P53 expression

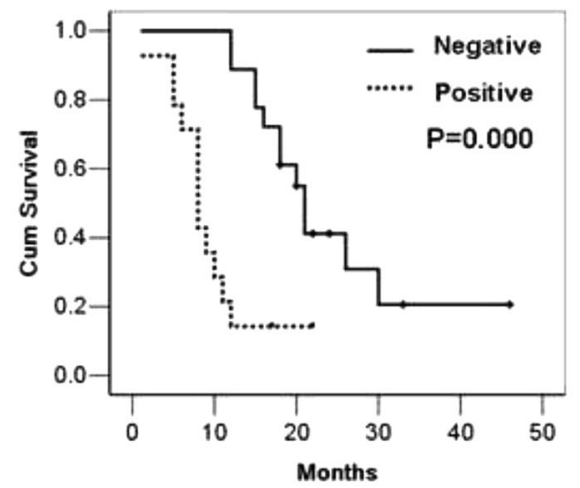

CA IX expression

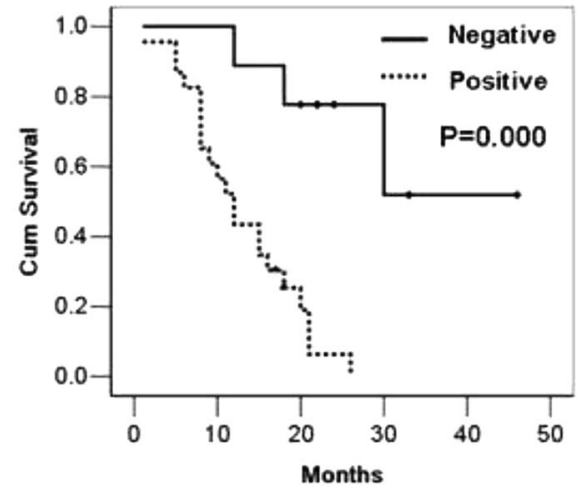

Bcl2 expression

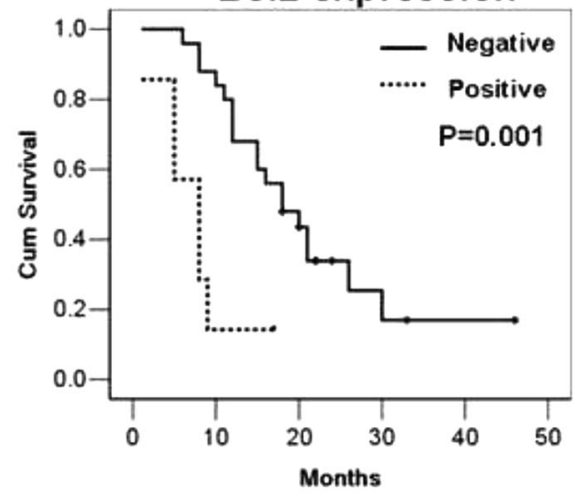

Fig. 3 The Kaplan-Meier survival curves comparing overall survival according to the expressions of CD10, CAIX, p53 and BCl-2. Univariate and multiple analyses showed that the positive expressions of CAIX, p53 and BCl-2 were all adverse prognostic factors for tumor related survival

molecular weights to identify the epithelial expression patterns of sarcomatoid cells.

\section{Mesenchymal markers}

As reported in the literature most sarcomatoid cells expressed vimentin with the positive rates of $56 \%-100 \%$ [5], few cases expressed actin and S100 [6]. In the present study, all 42 cases showed strong positive expression of vimentin in sarcomatoid cells. Among them, 30 cases expressed epithelial markers, 8 cases expressed SMA, but none of them expressed S100. Statistical analysis revealed the expression of vimentin in carcinoma and sarcomatoid cells was significantly different, which might be due to the lack of vimentin expression in 4 ChRCCs. Notably, vimentin was expressed in the sarcomatoid cells of CCRCC, which is different from that of ChRCC.

\section{RCCs specific markers}

CAIX is a valuable marker indicating low endogenous oxygen level, which catalyzes the formation of carbonic acid from carbon dioxide and adjusts the $\mathrm{pH}$ value in tumor cells to adapt to the surrounding microenvironment and facilitate tumor proliferation. CAIX is now considered as a highly sensitive and specific marker for clear cell renal cell carcinoma [7]. Immunohistochemical study on CAIX in RCC with sarcomatoid differentiation has been rarely reported up till now. We revealed a high frequency of expression of CAIX in sarcomatoid cells (90\%) with extensive and strong staining. Moreover, strong CAIX staining in sarcomatoid cells had been observed regardless the negative expression of CAIX in carcinoma cells (5 CCRCCs, 6 ChRCCs), indicating its involvement in proliferation of sarcomatoid cells and the transformation of carcinoma to sarcomatoid cells. It is known that CD10 could be used as a supplementary diagnostic marker for renal cell carcinoma. 32 cases showed strong CD10 expression in sarcomatoid cells. Among them, CD10 was expressed in both carcinoma and sarcomatoid cells in 20 cases, whereas 12 cases presented CD10 expression only in sarcomatoid cells. PAX8 belongs to PAX gene family, which is an important transcription factor in renal organogenesis and a reliable marker for primary kidney cancer [7]. Chang et al. [8] had reported that the expression rates of PAX8 were $69 \%$ and $18 \%$ in sarcomatoid cells of RCC with sarcomatoid differentiation and sarcomatoid urothelial carcinoma, respectively. However, it was rarely expressed in renal epithelioid 
angiomyolipoma and leiomyosarcoma, suggesting that it could be used as a good diagnostic marker for RCC with sarcomatoid differentiation. The present study revealed that PAX8 was expressed in carcinoma cells of 34 cases and sarcomatoid cells of 27 cases. Among them, 24 cases showed extensive expression in both two components, which leads to an idea that PAX8 could be used as a useful diagnostic marker for identifying the RCC with sarcomatoid differentiation in fine needle aspiration cases and in metastatic RCC with sarcomatoid differentiation. AMACR was rarely expressed in sarcomatoid cells, and only 6 cases showed strong AMACR expression in our study.

\section{Prognosis and treatment related markers}

CAIX, one of the most important tumor-associated carbonic anhydrases (CAs), is strongly induced by hypoxia in renal carcinoma cells, which could promote the growth of tumor cells. However, few research endeavors have been devoted to studying its role in predicting the outcome of RCC with sarcomatoid differentiation. Tickoo et al. [9] reported that over expression of CAIX showed no association with survival in 22 clear cell RCCs and 12 nonclear cell RCCs with sarcomatoid differentiation. Whereas, the results of the present study indicated that positive expression of CAIX was related to the adverse prognosis of 42 cases of RCCs with sarcomatoid differentiation. Therefore, more cases are needed for further identifying the predictive function of CAIX for the prognosis of RCCs with sarcomatoid differentiation.

P53 is a tumor suppressor gene, which plays a key role in carcinogenesis. Our study demonstrated that the expression rate of p53 was $48 \%$ in sarcomatoid cells, which is higher than that in carcinoma cells, suggesting that p53 might be involved in triggering the development of high malignant sarcomatoid tumors from renal cell carcinoma. Furthermore, p53 could be a reliable marker for predicting the prognosis or treatment outcome of RCC with sarcomatoid differentiation.

Bcl-2 is an apoptosis suppressor gene; therefore its overexpression is a hallmark of cell proliferation and suppression of cell apoptosis. Our result showed a significant difference of $\mathrm{Bcl}-2$ expressions between carcinoma cells and sarcomatoid cells, suggesting that Bcl-2 might facilitate the aberrant growth of carcinoma cells and the transformation of carcinoma cells to high malignant sarcomatoid differentiation via suppression of apoptosis. In addition, the expression of Bcl- 2 was associated with the adverse outcome of RCC with sarcomatoid differentiation and $\mathrm{Bcl}-2$ could be an important prognostic factor for the overall survival in RCC with sarcomatoid differentiation.

Additionally, Castillo et al. [10] had reported that sarcomatoid cells in $94.7 \%$ of RCC with sarcomatoid differentiation overexpressed CD117, suggesting the idea that tyrosine kinase inhibitor could be used for the therapy of CD117 positive RCC with sarcomatoid components patients. Wang et al. [11] did not find any CD117 expression in sarcomatoid urothelial carcinoma of the upper urinary tract. Our results showed CD117 only expressed in sarcomatoid cells of 3 cases of RCC and carcinoma cells of 7 ChRCCs with sarcomatoid differentiation. More studies are needed to discover the possible therapeutic use of tyrosine kinase inhibitor for RCC with sarcomatoid differentiation.

Due to the rarity of RCCs with sarcomatoid differentiation, more cases are needed to further confirm the specific features of the expression panels of these 19 immunomarkers, especially the prognostic values of CAIX, p53 and $\mathrm{Bcl}-2$ playing in RCCs with sarcomatoid differentiation.

\section{Conclusions}

Sarcomatoid cells in RCC with sarcomatoid differentiation express both epithelial and mesenchymal markers, supporting their epithelial origin. The difference of expression profiles between carcinoma cells and sarcomatoid cells might be due to the distinct orientation and the degree of differentiation in renal cell carcinoma. CAIX, p53 and bcl-2 might play important roles in triggering the transformation from renal cell carcinoma to high malignant sarcomatoid component, and could be beneficial to predicting the prognosis or treatment outcome of RCC with sarcomatoid differentiation. PAX8, CAIX and CD10 could be used as the reliable markers to determine the renal origin of sarcomatoid cells, especially in fine needle aspiration cases and the cases of metastatic RCC with sarcomatoid differentiation. The clinical therapeutic application of tyrosine kinase inhibitors to RCC with sarcomatoid differentiation needs to be investigated with more research studies and clinical trials.

\section{Abbreviations}

AMACR: Alpha-methylacylCoA racemase; CCRCC: Clear cell renal cell carcinoma; ChRCC: Chromophobe renal cell carcinoma; RCC: Renal cell carcinoma

\section{Acknowledgements}

Not applicable.

\section{Funding}

This work was supported by National Natural Science Foundation of China (81201654) in the design of the study and in writing the manuscript. Shandong Province Science and Technology Development Plan (2013GSF11866) and Medical Research Guiding Program of Qingdao (2014WJZD195) contributed in collection, analysis, and interpretation of data.

\section{Availability of data and materials}

All data generated or analysed during this study are included in this published article.

\section{Authors' contributions}

WY was involved in drafting the manuscript and revising it critically for important intellectual content. YW made substantial contributions to acquisition of data. YJ analysis and interpretation of data. WZ was involved in drafting the manuscript or revising it. YL made substantial contributions to conception and design of the study. All authors read and approved the final manuscript. 


\section{Competing interests}

The authors declare that they have no competing interests.

\section{Consent for publication}

Not applicable.

\section{Ethics approval and consent to participate}

The use of human subjects in the study has been approved by the Ethics Committee of the Affiliated Hospital of Qingdao University. The patients consented to participate in the study by verbal informed consent. The need for written consent has been waived by the Ethics Committee of the Affiliated Hospital of Qingdao University, and the Ethics Committee approved the verbal informed consent.

\section{Publisher's Note}

Springer Nature remains neutral with regard to jurisdictional claims in published maps and institutional affiliations.

\section{Author details}

'Department of Pathology, The Affiliated Hospital of Qingdao University, 16 Jiangsu Road, Qingdao 266003, China. ${ }^{2}$ Department of Pathology, 401 Hospital of People's Liberation Army, 22 Minjiang Rd, Qingdao 266071, China. ${ }^{3}$ Department of Vascular Surgery, The Affiliated Hospital of Qingdao University, Qingdao 266003, China.

Received: 16 February 2016 Accepted: 11 April 2017

Published online: 27 April 2017

\section{References}

1. Eble JN, Sauter G, Epstein J, et al. World Health Organization classification of tumours of the urinary system and male genital organs. Lyon, France: IARC Press. 2004

2. Moch $H$, Humphrey PA, Ulbright TM, et al. WHO classification of tumors of the urinary and male genital organs. Lyon, France: IARC Press, 2016.

3. Pamela A, Arnoux V, Long JA, et al. Sarcomatoid renal cell carcinoma: follow-up of a series of 23 patients. Prog Urol. 2014;24(5):301-6.

4. Lopez-Beltran A, Scarpelli M, Montironi R, et al. 2004 WHO classification of the renal tumors of the adults. EurUrol. 2006; 49 (5): 798-805.

5. Yan Y, Liu L, Zhou J, et al. Clinicopathologic characteristics and prognostic factors of sarcomatoid renal cell carcinoma. J Cancer Res Clin Oncol. 2015;141(2):345-52.

6. Gadre SA, Math SK, Elfeel KA, et al. Cytology of a Sarcomatoid renal cell carcinoma with unusual Coexpression of S-100 protein: a case report, review of the Literatureand Cytologic-Histologic correlation. Diagn Cytopathol. 2009;37(3):195-8.

7. Zhang W, Yu W, Xia Y, et al. The expressions of CAIX, PAX2 and PAX8 in epithelial renal tumors and the clinicopathological significance. Zhonghua Bing Li Xue Za Zhi. 2013:42:442-5.

8. Chang A, Brimo F, Montgomery EA, et al. Use of PAX8 and GATA3 in diagnosing sarcomatoid renal cell carcinoma and sarcomatoid urothelial carcinoma. Hum Pathol. 2013;44(8):1563-8.

9. Tickoo SK, Alden DS, Fine S, et al. Immunohistochemical expression of hypoxia inducible factor-1alpha and its downstream molecules in sarcomatoid renal cell carcinoma. J Urol. 2007;177(4):1258-63.

10. Castillo M, Petit A, Mellado B, et al. C-kit expression in sarcomatoid renal cell carcinoma: potential therapy with imtinib. J Urol. 2004;171(6 Pt 1):2176-80.

11. Wang X, MacLennan GT, Zhang S, et al. Sarcomatoid carcinoma of the upper urinary tract: clinical outcome and molecular characterization. Hum Pathol. 2009:40(2):211-7.

\section{Submit your next manuscript to BioMed Central and we will help you at every step:}

- We accept pre-submission inquiries

- Our selector tool helps you to find the most relevant journal

- We provide round the clock customer support

- Convenient online submission

- Thorough peer review

- Inclusion in PubMed and all major indexing services

- Maximum visibility for your research

Submit your manuscript at www.biomedcentral.com/submit 